Final version published in:

J.M. Rodríguez, M. Castilla, J.D. Álvarez, F. Rodríguez y M. Berenguel (2015). “A Fuzzy Controller for Visual Comfort inside a Meeting-Room". En: 23rd Mediterranean Conference on Control and Automation (MED), Torremolinos (Spain).

DOI: $\underline{10.1109 / M E D .2015 .7158888}$

http://ieeexplore.ieee.org/xpls/abs all.jsp?arnumber $=7158888$ 


\title{
A Fuzzy Controller for Visual Comfort inside a Meeting-Room
}

\author{
J.M. Rodríguez, M. Castilla, J.D. Álvarez, F. Rodríguez and M. Berenguel
}

\begin{abstract}
In general, human beings receive most part of information through eyesight. Therefore, light is an essential element which allows people to distinguish the shape, colour and perspective of the environment around them. Moreover, energy efficiency in buildings is a problem that is being widely analysed since energy consumption inside them represents approximately $40 \%$ of total world energy consumption. In addition, illumination supposes around $20 \%$ of total energy consumption in houses and between $40 \%$ and $70 \%$ in shops and offices. This paper presents a fuzzy logic controller which allows maintaining visual comfort inside a meeting-room through an efficient use of both natural and artificial lights. In order to probe the effectiveness of the proposed control system, suitable real results obtained in a meeting-room of the CIESOL bioclimatic building are included and commented.

Index Terms - Visual comfort; Fuzzy logic control
\end{abstract}

\section{INTRODUCTION}

According to recent studies, lighting represents between $40 \%$ and $70 \%$ of total energy consumption within the tertiary sector, and moreover, it is expected that this consumption will increase in the future [1]. Therefore, it is necessary to develop some strategies which allow to reduce this energy consumption without interfering on users' visual comfort.

Visual comfort inside a certain environment is highly related with the indoor illuminance level. Moreover, illuminance level depends on two essential factors: daylight and artificial lighting. Hence, visual comfort can be achieved by combining, in an efficient way, the use of both daylight and artificial lighting. To do that, there are different kinds of shading elements to regulate the amount of daylight inside the environment, such as venetian blinds or rolling shutters, and regulative artificial lighting. In addition, to reach these objectives, that is to maintain visual comfort and to reduce the amount of energy derived from it, the use of appropriate control systems is a widespread strategy. These control techniques range from classical control approaches [2] to intelligent controllers based on fuzzy logic [3], [4], [5], [6], or even a combination of them [7].

The main aim of this paper is to present a fuzzy logic control approach that ensures visual comfort inside the meeting-room of a bioclimatic building in an efficient way. However, the application of a fuzzy logic control strategy is not limited to this field, and thus, it is possible to find numerous works which apply this control strategy to different areas. For instance, in [8] it is used within the education field to teach advanced control engineering in a experimental

The authors are with University of Almería Agrifood Campus of International Excellence, ceiA3, CIESOL, Joint Center University of Almería - CIEMAT and Automatic Control, Robotics and Mechatronics Research Group, Ctra. Sacramento s/n, 04120 La Cañada (Almería), España (mcastilla, jhervas, frrodrig, beren) dual.es manner. In the case of study presented in this paper, the performance of this strategy has been evaluated by means of real tests inside a meeting-room. The results obtained in this paper can be easily extrapolated to any room with a suitable network of sensors and motorised blinds and artificial lighting.

The paper is organised as follows: Section II introduces the concept of visual comfort and performs a brief introduction to the indexes which have been used to its estimation. In Sec. III, the fuzzy logic control architecture is explained, and a short description of the meeting-room and the illuminance model used with the proposed control architecture is performed. Section IV is devoted to the obtained real results. Finally, in Sec. V, the main conclusion and future works are summarised.

\section{VISUAL COMFORT}

The sense of sight is a cornerstone in the processing of information by humans since it allows them to perceive the colour, form and perspective of the different elements in a certain environment [9]. Hence, a correct lighting of any indoor environment should meet the following requirements [10]: i) To guarantee the security of people inside it; ii) to provide an appropriate lighting level for the development of visual works; and iii) to assist in the creation of an adequate visual surrounding.

Furthermore, in accordance with international standard UNE EN-12665 [11] visual comfort can be defined as $a$ subjective condition of visual well-being induced by the visual environment. From this definition, it can be established that even though there is a psychological factor closely related with visual comfort, it is possible to perform its evaluation through some physical properties of the visual environment [12]. More specifically, a visual comfort condition can be defined as a function of the luminance distribution, illuminance and its distribution, glare, colour of light, colour rendering, flicker rate and amount of daylight [13]. The ideal values for the previous parameters are estimated in international research and collected by means of international standards as UNE EN-12464-1 [14]. For instance, in Table I can be observed the appropriate levels of indoor illuminance, glare rating and colour rendering for common tasks which are developed inside offices.

In this work, visual comfort has been estimated as a function of appropriate levels of both illuminance and glare. In this section, some indexes for these parameters are going to be defined and the methodology used to calculate them will be explained. 
TABLE I

RECOMMENDATIONS FOR INDOOR LIGHTING OF OFFICES [10]

\begin{tabular}{|c||c||c|}
\hline Activity & $\begin{array}{c}\text { Maintained } \\
\text { illuminance } \\
{[\text { lux] }}\end{array}$ & $\begin{array}{c}\text { Limiting } \\
\text { glare rating }\end{array}$ \\
\hline Filing, copying, etc. & 300 & 19 \\
\hline Writing, typing, reading, etc. & 500 & 19 \\
\hline Technical drawing & 750 & 16 \\
\hline CAD work stations & 500 & 19 \\
\hline Conference and meeting-rooms & 500 & 19 \\
\hline Archives & 200 & 25 \\
\hline
\end{tabular}

\section{A. Illuminance level}

Illuminance level inside a certain environment is an essential parameter in order to evaluate visual comfort. The appropriate illuminance levels are established depending on the type of environment and the activity developed inside it. In this paper, to evaluate visual comfort as a function of the illuminance level an index called $D I L L$ has been used. This index represents the difference between real and desired illuminance levels, see (1).

$$
D I L L=I L L_{\text {real }}-I L L_{\text {des }}
$$

where $I L L_{\text {real }}$ and $I L L_{\text {des }}$ are the real and desired illuminance levels expressed in [lux].

\section{B. Glare}

Glare is that condition of vision in which there is discomfort mainly originated by an inadequate distribution or range of indoor illuminance, or to extreme contrasts [10]. In this work, to estimate visual discomfort due to glare the Unified Glare Rating (UGR) [14] index has been used. This index expresses the discomfort glare caused by the presence of bright light sources, that is, luminaries or windows [15]. This index is calculated just as it can be observed in (2). The results provided by UGR index are within the range of $13-30$, and moreover, the higher UGR index values, the bigger visual discomfort condition.

$$
\begin{gathered}
U G R=8 \log _{10}\left(\frac{0.25}{L_{b}} \sum_{i=1}^{N} \frac{L_{s_{i}}^{2} \omega_{i}}{p_{\text {Guth }}^{2}}\right) \\
D U G R=U G R_{\text {real }}-U G R_{\text {des }}
\end{gathered}
$$

In the previous equations $L_{b}$ is the background luminance in $\left[\mathrm{cd} / \mathrm{m}^{2}\right], N$ represents the number of luminaries inside the room, and $L_{s_{i}}, \omega_{i}$ and $p_{G u t h_{i}}$ are the luminance in $\left[c d / m^{2}\right]$, the solid angle subtended at the observers eye and the Guth position index provided by the $i^{t h}$ luminary, respectively. In this work, glare has been estimated using DIALux evo 3 software [16]. More information about the procedure to calculate this index can be found in [13].

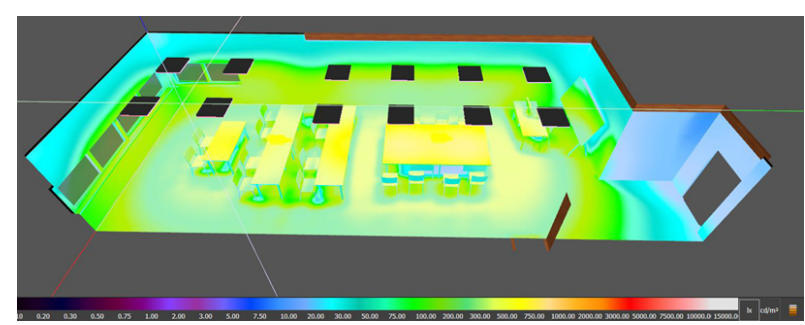

Fig. 1. 3D representation of indoor illuminance inside the meeting-room provided by artificial lights. Simulation performed using DIALux evo 3 software [16]

\section{CONTROL SYSTEM ARCHITECTURE}

As it was mentioned previously, visual comfort inside a certain environment depends of two basic factors: illuminance level and glare. These factors are estimated by means of the DILL and DUGR indexes respectively. In addition, the illumination level can be controlled through the regulation of the amount of natural and artificial light in the environment. Therefore, it is necessary to look for a trade-off between them in order to achieve an appropriate illuminance level and, at the same time, increase energy efficiency. To do that, it is very common both the use of shading elements, just as venetian blinds, and adequate control techniques to regulate artificial light

\section{A. Scope of the research: A meeting-room}

The control architecture proposed in this paper has been evaluated by means of real tests inside a meeting-room of the CIESOL bioclimatic building. The CIESOL building is a solar energy research centre located inside the Campus of the University of Almería, in the south-east of Spain. Concretely, the meeting-room with a total surface of $13.37 \times 5 \mathrm{~m}^{2}$ is located on the first floor of the building with south orientation. It has three windows which are placed on the south and west façades with its associated inside venetian blinds. Therefore, by its distribution, in this work it has been considered that the room is divided into two different zones: projector zone and windows zone. Moreover, it counts with a pendant-mounted artificial lighting system consisting of 12 luminaries containing each one four TL-D 18W/54-765 Philips fluorescent lamps. In the projector zone are included 8 luminaries and in the windows zone there are 4 luminaries which assure an average illuminance level at the workspace of 500 lux, see Fig. 1.

Furthermore, this room has different actuators and sensors, see Fig. 2. On the one hand, the meeting-room counts with two indoor low-cost luxometers which are placed in a middle point of each zone, an outdoor luxometer located at the meteorological station of the building and a presence sensor to detect when the room is empty [17]. On the other hand, the set of actuators is composed by the motors of the blinds which allow to control the venetian blinds degree of aperture and orientation and an artificial light switch (On/Off actuator) for each zone. 


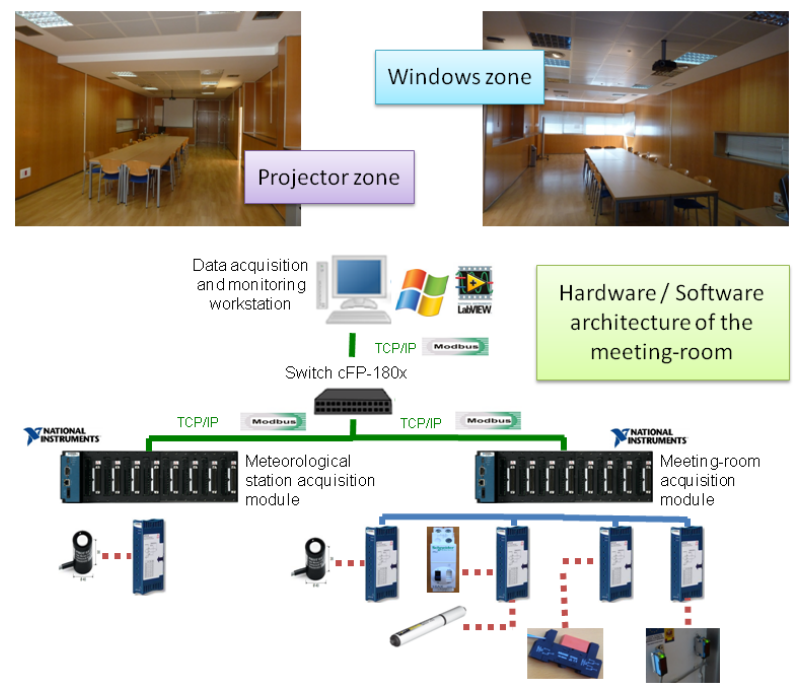

Fig. 2. Meeting-room zones and hardware architecture

\section{B. Modelling of the illuminance level}

The meeting-room which has been used to test the proposed control strategy has two windows with south orientation and one window which faces west. Therefore, in order to develop an appropriate control strategy, a model which takes into account both natural and artificial light and also the Sun position has to be developed. There exists reliable packages allowing to simulate or estimate illuminance, for instance the same used for glare estimation (DIALux evo 3 software [16]). In order to evaluate such model, tests have been performed to compare the obtained illuminance level with that provided by that package. This model can also be used for control design purposes. Within the framework of fuzzy logic control, a very simple model has also been obtained helping to define the rules used by the controller, as will be explained in what follows. The following assumptions have been taken into account:

- Mainly due to the characteristics of the meeting-room it has been divided into two different zones: the projector zone (without windows) and the windows zone.

- Artificial lighting which is installed inside the meetingroom has an ON/OFF nature, and moreover, it is independent for each zone.

- Although the blinds of the meeting-room can be considered as a continuous actuator, six different positions for the aperture $\left(A P_{x}\right)$ and orientation $\left(O R_{x}\right)$ in percentage of each one of the blinds, \{Totally closed (TC), Almost closed (AC), Medium-Low (ML), Medium (M), Open (O), Totally open (TO)\}, have been defined, see Table II. These positions cover the combinations which have a representative influence on indoor illuminance.

Due to the quantization errors induced by the low-cost luxometers, as a preliminary approach, a static model in the form of tables relating the position of the actuators to the illuminance gain has been used. The values in this table obviously depend also on the Sun position, that can be described by its declination and solar azimuth angle values (which can be easily computed from time and location data [18], [19], [20]), considering a discrete grid of the range of variation of such values. In this case, due to the intrinsic errors induced by sensors and the limited amount of tests carried out at present, a set of only 30 tables covering a limited range of Sun positions and actuators states has been obtained, providing acceptable results when used for control design purposes. New sensors are going to be installed so that reliable data for identification purposes will be obtained, allowing to develop a better fuzzy inference table or even a static neural network (or a dynamic one if the dynamics can be captured by the new sensors) using as inputs the state of the actuators and the discretized sun position and providing a more accurate prediction of the illuminance level that can be used for developing more accurate controllers.

TABLE II

DESCRIPTION OF THE POSITIONS FOR THE BLINDS OF THE MEETING-ROOM

\begin{tabular}{|c||c||c||c||c||c||c|}
\hline Position & $A P_{1}$ & $A P_{2}$ & $A P_{3}$ & $O R_{1}$ & $O R_{2}$ & $O R_{3}$ \\
\hline TC & 0 & 0 & 0 & 0 & 0 & 0 \\
\hline AC & 0 & 0 & 0 & 50 & 0 & 0 \\
\hline ML & 50 & 50 & 0 & - & - & 50 \\
\hline M & 100 & 0 & 0 & - & 0 & 100 \\
\hline O & 100 & 0 & 0 & - & 100 & 100 \\
\hline TO & 100 & 100 & 100 & - & - & - \\
\hline
\end{tabular}

As has been mentioned, different tests under several conditions have been performed along 2014. For instance, in Figs. 3 and 4 one of these tests is shown. More specifically, this test was realized in a clear summer afternoon under several configurations of lights and blinds (aperture and orientation), see Fig. 3. Moreover, the measured indoor illuminance at both projector and windows zone is shown in Fig. 4. Afterwards, an illuminance model for each zone has been obtained. The results have been also compared with those provided by DIALux evo 3 software.

\section{A Fuzzy Logic Control System}

The main objective of the proposed control architecture, see Fig. 5, is to maintain visual comfort within appropriate limits in an efficient way. Therefore, the outputs of the controlled process are the real illumination level $(I L L)$ and glare (Glare) which have been measured and estimated inside the meeting-room, respectively. Consequently, the proposed fuzzy logic controller has as inputs the DILL and DUGR indexes which have been defined in Sec. II and the current state of the blinds and lights of the meeting-room, STBlinds and ST Lights respectively. On the other hand, the outputs of the fuzzy logic controller are the appropriate control signals for each one of the venetian blinds $(B L I N D S)$ and the artificial lights $(L I G H T S)$. Nevertheless, as the physical values of the input variables may differ significantly in magnitude, it is necessary to perform an input preprocessing process that will allow scaling the input variables, just as it is shown in (4) and (5). 

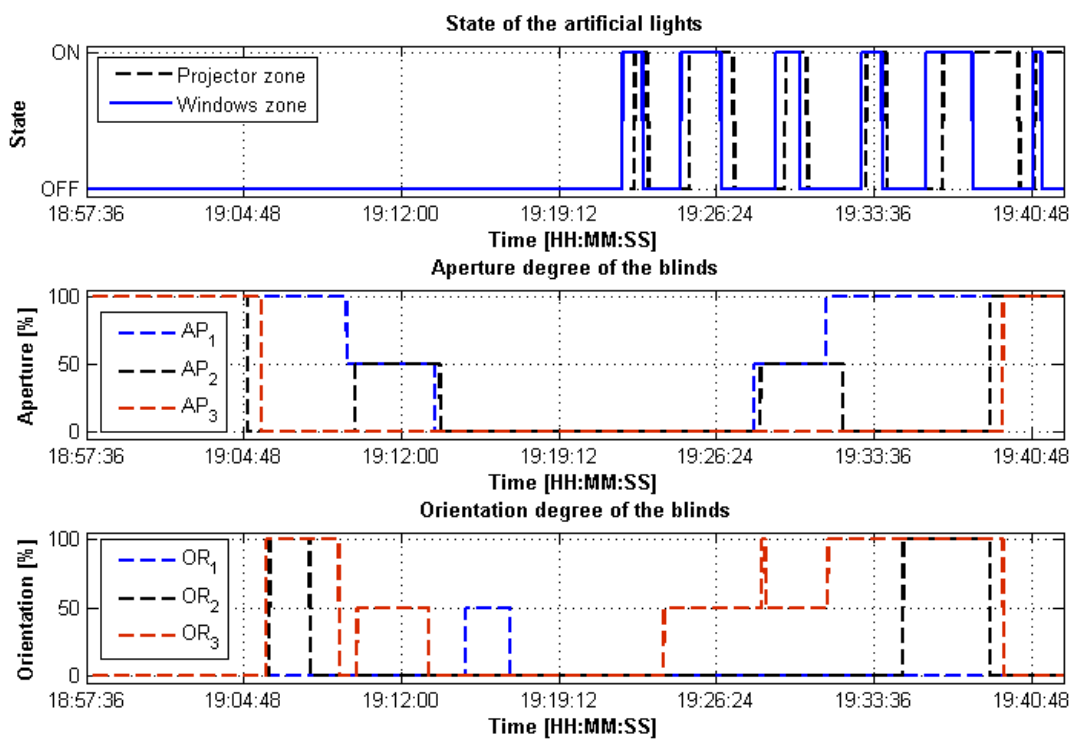

Fig. 3. Example of signals used on the actuators for the identification procedure
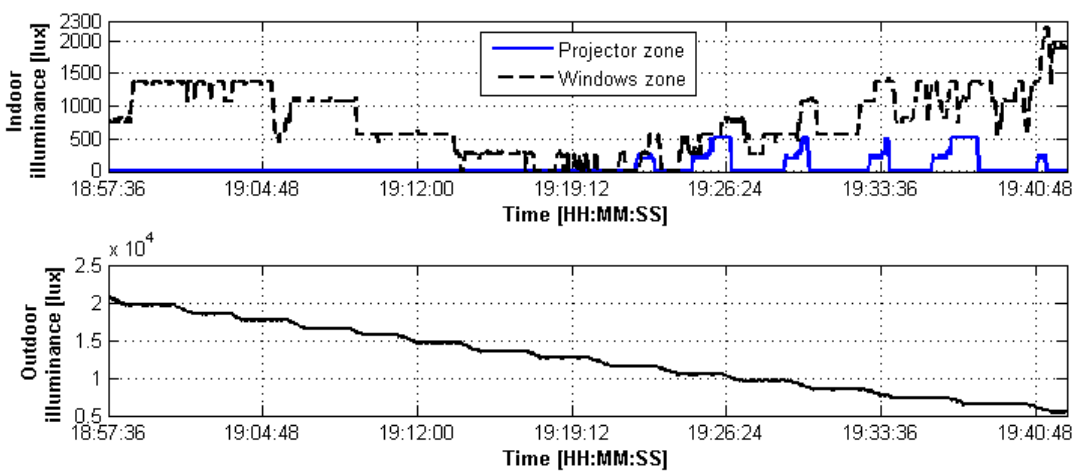

Fig. 4. Example of measured indoor and outdoor illuminance for the identification procedure

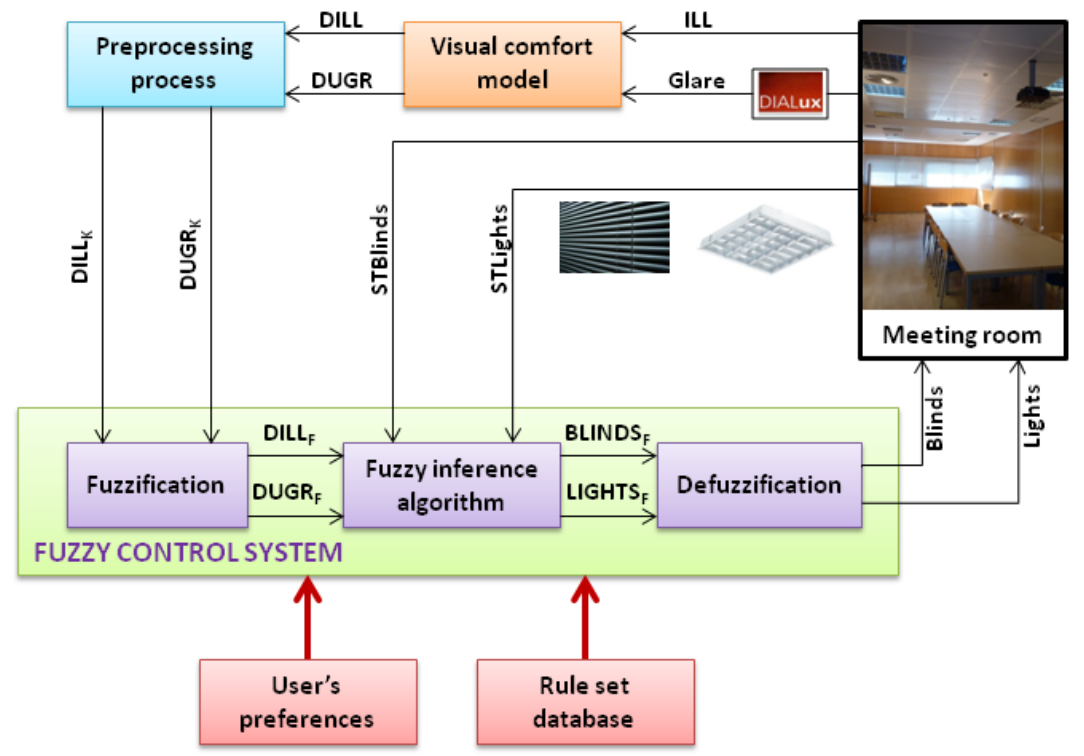

Fig. 5. Architecture of the Fuzzy Logic Control System 


$$
\begin{gathered}
D I L L_{K}=\frac{I L L_{\text {real }}-I L L_{\text {des }}}{K_{D I L L}} \\
D U G R_{K}=\frac{U G R_{\text {real }}-U G R_{d e s}}{K_{D U G R}}
\end{gathered}
$$

In the previous equations, $K_{D I L L}$ and $K_{D U G R}$ are parameters used to delimit DILL and DUGR indexes among $[-1,10]$ and $[-6,5]$, to follow the same approach presented in [4].

Furthermore, these output signals are estimated by means of a fuzzy inference algorithm which uses suitable fuzzy sets represented by memberships functions, a rule set database and provides as a result others fuzzy sets [21]. Figure 5 shows the main blocks describing the fuzzy logic controller: the block fuzzifier, the control block (fuzzy rule base and inference procedure) and the defuzzifier block.

On the one hand, the fuzzification process maps the scaled input and output variables into appropriate fuzzy sets [21]. More specifically, in this case the $D I L L_{K}$ and $D U G R_{K}$ input variables have been transformed into two fuzzy variables consisting of the fuzzy sets defined by Negative, Small, Medium, Large, Too Large $\}$ and Imperceptible, Acceptable, Uncomfortable $\}$ respectively. These input fuzzy variables represent the size of the existing difference between the real and desired value of each index. Moreover, in this work, triangular memberships functions defined by (6) have been used as it is shown in Fig. 6. In addition, two different output fuzzy variables have been established $B L I N D S_{F}$ and $L I G H T S_{F}$. These variables consist of the fuzzy sets defined by $\{$ Totally closed, Almost closed, Medium-Low, Medium, Open, Totally open $\}$ and $\{$ On, Off $\}$ respectively. Besides, the previous output fuzzy sets have been defined according to the blind positions described within the modelling section and the On/Off artificial light actuator. Nevertheless, in this case singleton memberships functions defined by (7) have been used (notice that in order to represent the surfaces represented in Figs. 7 and 8, triangular membership functions with very narrow base have been used).

$$
\begin{gathered}
\mu_{\text {In }}(x)= \begin{cases}\frac{x-a}{b-a}, & a \leq x \leq b ; \\
\frac{c-x}{c-b}, & b<x \leq c ; \\
0, & x<a \text { or } x>c\end{cases} \\
\mu_{\text {Out }}(x)= \begin{cases}1, & x=x^{*} ; \\
0, & \text { otherwise }\end{cases}
\end{gathered}
$$

On the other hand, the defuzzification process transforms the information provided by the inference algorithm into different control signals which can be applied to the actuators [21]. To do that, the weighted average (centroid for narrow base triangular membership functions) method [21] has been used.

The fuzzy inference algorithm is responsible of the estimation of a fuzzy output as a function of a certain fuzzy input pattern. It has been proposed as a rule based fuzzy algorithm and thus, it is necessary to define rule set database which should include the expert knowledge of the controlled system, acquired in this case from the performed tests and
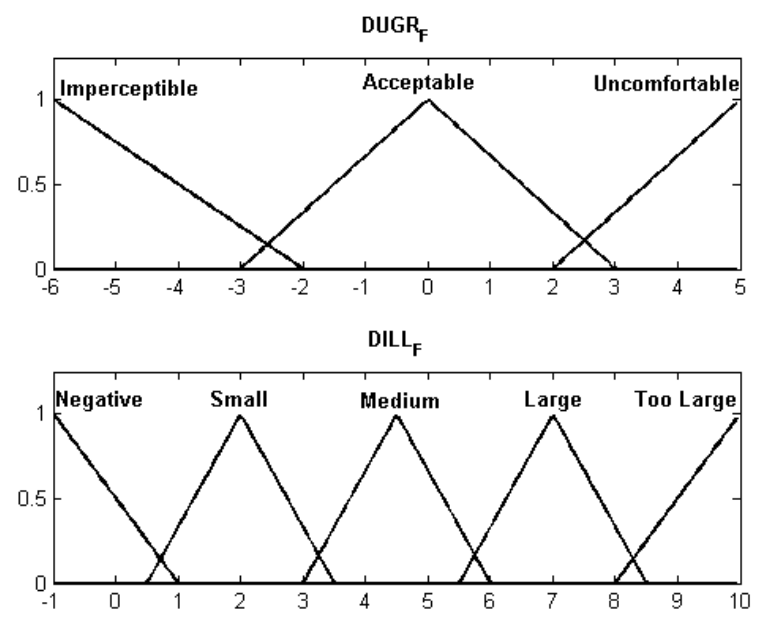

Fig. 6. Membership functions of the fuzzy input variables
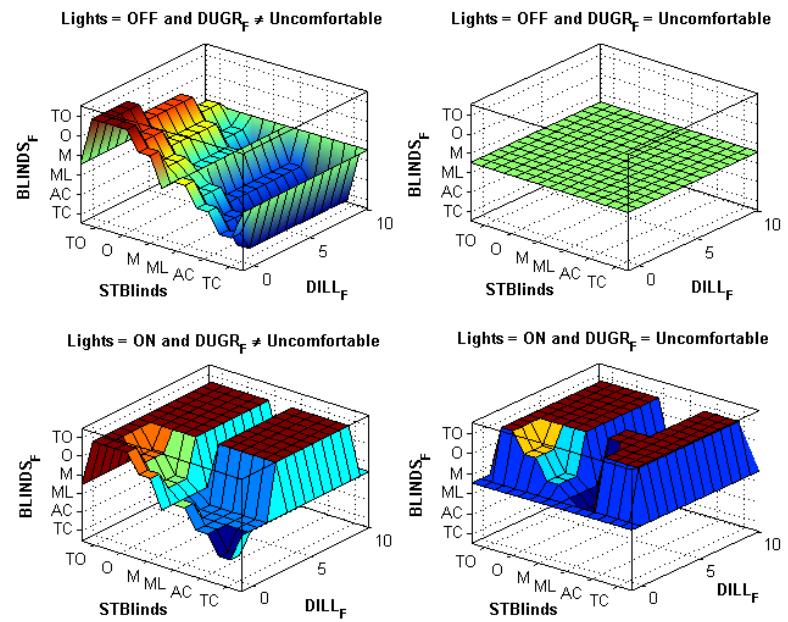

Fig. 7. Output signal for $B L I N D S_{F}$ variable from the fuzzy inference algorithm (these simulations have been performed using Fuzzy Logic Toolbox of Matlab [22])

using both the DIALux model and the table-based simplified model described in Section III-B leading to the definition of the set of rules governing the system behaviour. Therefore, the existing relationships among input/output variables have been represented by means of If-Then rules which are represented by a statement of the form "If an antecedent proposition, Then a consequent proposition" [21]. More specifically, in this work, 84 rules have been specified by means of Mandami models where both the antecedent and consequent are fuzzy propositions [21]. The set of rules which has been defined to solve the problem proposed in this work is graphically represented in Figs. 7 and 8 by means of the existing relationships among input and output fuzzy variables as a function of the rule set database. More specifically, Fig. 7 shows the relationships among the output fuzzy variable $B L I N D S_{F}$ and the input fuzzy variables which make reference to the illuminance level $\left(D I L L_{F}\right)$, and 

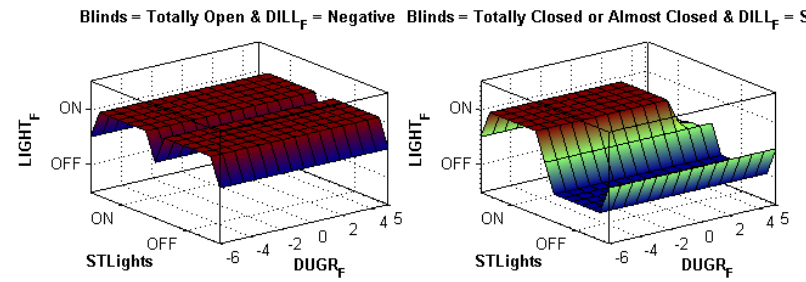

Blinds $\neq$ Totally Open \& $\mathrm{DILL}_{\mathrm{F}}=$ Negative $\mathrm{OR}$ Blinds $\neq$ Totally Closed or Almost Closed \& DIL $L_{F}=$ Small $\quad$ DILL $_{F}=$ Medium, Large or Too Large
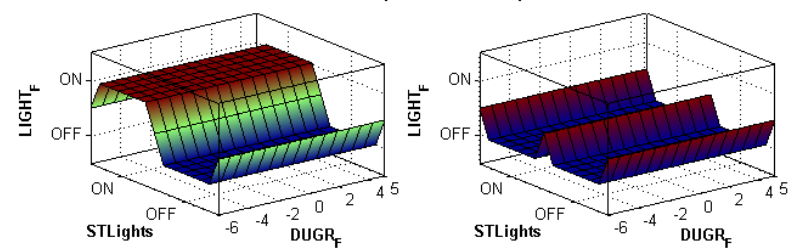

Fig. 8. Output signal for $L I G H T S_{F}$ variable from the fuzzy inference algorithm (these simulations have been performed using Fuzzy Logic Toolbox of Matlab [22])

the current state of the blinds (STBlinds) under different conditions of LIGHTS and DUGR . On the other hand, in Fig. 8 it is shown the relationships among the output fuzzy variable $L I G H T S_{F}$ and the input fuzzy variables which make reference to glare $\left(D U G R_{F}\right)$, and the current state of the lights (STLights) under different conditions of BLINDS and DILLF. These rules and the rest of parameters related with the proposed fuzzy logic controller have been determined heuristically as a function of the knowledge acquired of the controlled process through tests and models in Section III-B, and based on the work proposed by [4].

Finally, an extra condition has been added to the proposed control system, that is, it disconnects the artificial lights of the meeting-room when it is empty for a period of time bigger than $5 \mathrm{~min}$. Besides, this extra condition also avoids the execution of the fuzzy logic controller, and thus, the application of new control actions. To do that, a presence sensor installed in the meeting-room has been used. More specifically, it has been used combined with a timer as a trigger of a high-priority action which allows the temporary deactivation of the automatic control system until any person will be detected again in the room. In Fig. 9 a flow diagram of the proposed fuzzy logic controller including the extra condition which has just been mentioned can be observed.

\section{RESULTS AND DISCUSSION}

The proposed fuzzy logic control system has been evaluated through real tests inside the meeting-room of a bioclimatic building along summer period. As it was mentioned previously, two different zones have been considered within this environment, the projector and the windows zone. Therefore, several real tests have been performed under different conditions. In this paper, one test which shows the actuation of the proposed control system along a complete typical

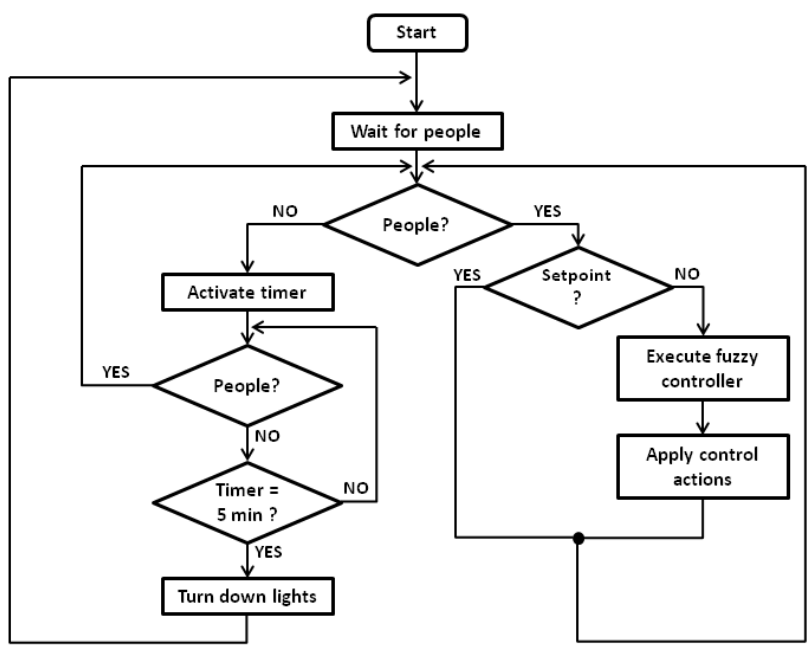

Fig. 9. Flow diagram of the proposed control system

working day without any forced condition has been selected and widely analysed.

The results obtained from this test can be observed in Fig. 10. This figure can be divided into six parts: the first row shows two graphs which represent the number of people inside the room, Fig. 10(a), and the outdoor illuminance, Fig. 10(b). In the second row, it can be observed the evolution of controlled variables (see Fig. 10(c)), that is, the indoor illuminance in both projector and windows zones and the reference which is established as a function of international standards and users preferences. Notice the offset produced in part by the low-cost luxometers used in this work, that are going to be replaced soon. Moreover, also in the second row, Fig. 10(d), it is shown the state of the artificial lights (ON/OFF actuators) in both zones. Finally, in the third row, it can be observed the state of each one of the venetian blinds of the meeting-room, that is their degree of aperture (Fig. 10(e)) and the orientation of their slats (Fig. 10(f)). Furthermore, glare conditions were analysed using Dialux simulation software [16] and the obtained results show that they were satisfied. Nevertheless, it is necessary to perform a detailed study in order to evaluate if glare conditions are satisfied along the whole year, as well as, the use of other indexes also considered by international standards such as the Daylight Glare Index (DGI) [13].

As it can be observed in Fig. 10(a), the working day begins around 9 a.m. when one person goes into the room. Afterwards, two more persons go into the meeting-room until $10.30 \mathrm{a} . \mathrm{m}$. when all of them leave the room for approximately half an hour. Then, at $13.00 \mathrm{p} . \mathrm{m}$. there was a meeting planned and five more persons arrive to this room. Hence, the illuminance reference is modified to a value equals to 300 lux since the projector mode is activated along the meeting. To do that, different modes which allow to modify the illuminance reference have been defined. In addition, from the acquisition and monitoring workstation it is possible that the administrator can change this setpoint. The meeting 
(a) Number of people

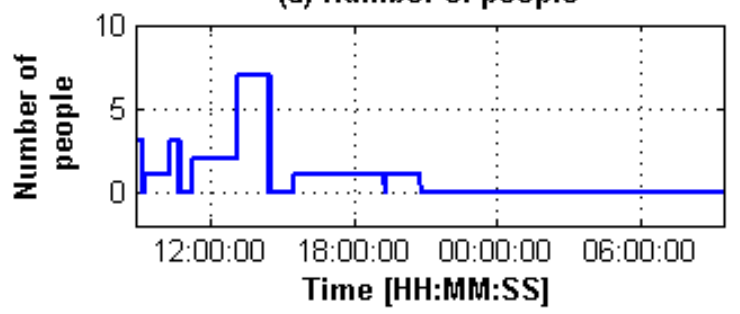

(c) Indoor illuminance

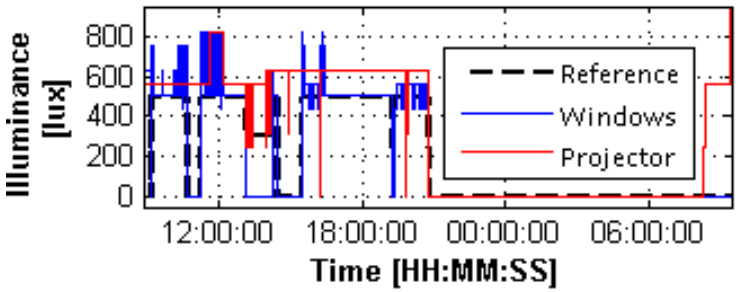

(e) State of the blinds: Aperture

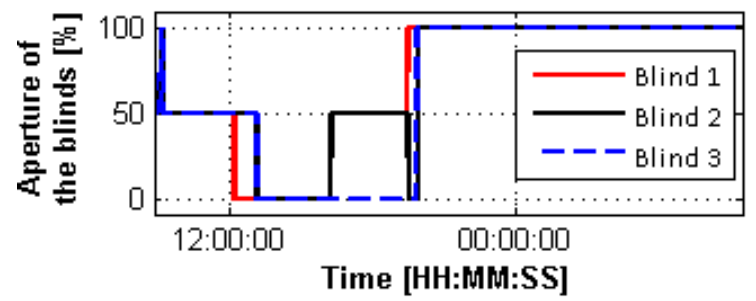

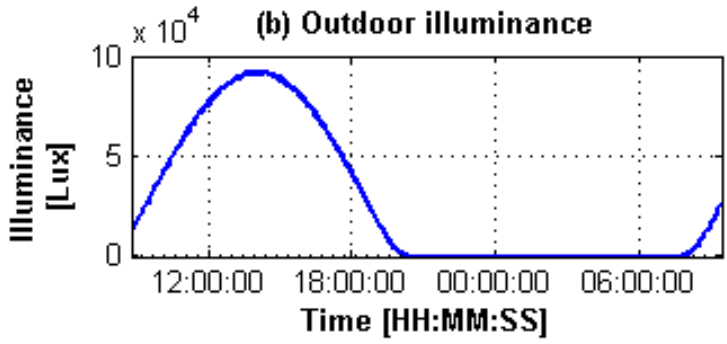

(d) State of the artificial lights

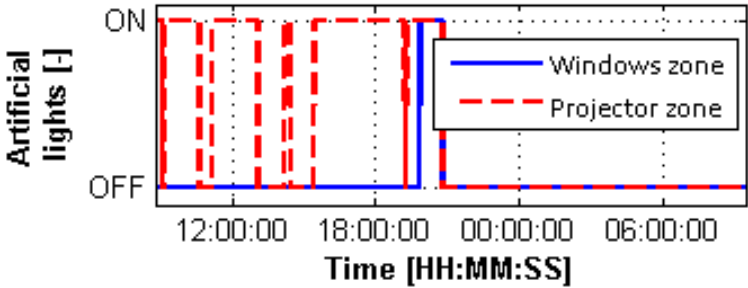

(f) State of the blinds: Orientation

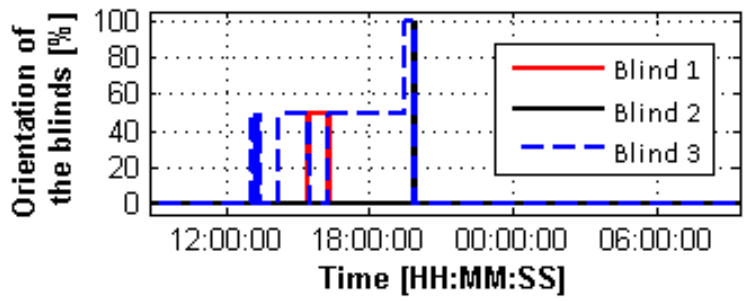

Fig. 10. Results obtained with the proposed fuzzy logic controller for a summer day

ends around 14.30 p.m. and all the users leave to have lunch. At 15.30 p.m one person returns to the meeting-room, and stay there all the afternoon. Along this period of time, it can be observed how, when it is getting dark, the fuzzy logic controller tries to maintain the established reference by firstly opening all the venetian blinds and when this strategy is not enough, turning on artificial lights. Finally, the work day finishes at 20.30 p.m. when the last person leaves the room until the next morning. At this moment, the control system turns off the artificial lights and maintains the venetian blinds at the same position.

To sum up, the obtained results show in Fig. 10(c) as the proposed control architecture is able to try to maintain the established reference in both zones (with offset due to the kind of sensors used), and under different conditions. Furthermore, Figs. 10(c)-(f) collect how people activity affects to the control system since artificial lights are turned down both when the room is empty or the projector mode is activated along the meeting (see Fig. 10(d)). It can also be seen that the fuzzy logic controller only actuates over the venetian blinds when the room is not empty, see Figs. 10(e) and (f). Finally, from the obtained results, it can be inferred that the proposed control system behaves correctly under the different situations which can be found in a typical working day inside a meeting-room.

\section{CONCLUSIONS AND FUTURE WORKS}

The availability of enough light is an essential point to reach appropriate working conditions. Besides, and mainly due to the current energy related regulations, another important factor is the optimisation of the use of energy inside buildings. To do that, it is necessary to combine innovations in the structural designs of buildings which make the most of natural light with appropriate control approaches. This paper is focused on the development of a preliminary fuzzy logic control system which allows maintaining visual comfort conditions and its validation inside a meeting-room. More specifically, this control system has been tested through real tests inside a meeting-room of a bioclimatic building. These preliminary results have shown as the proposed control architecture has been able to maintain the specified visual comfort conditions with the available means and, at the same time, decreasing the use of artificial light.

As future works, real tests with the proposed control architecture will be performed along different seasons, and moreover, it will be compared with other existing control approaches. In addition, other indexes for glare evaluation will be considered as the DGI index. Changes in the actual configuration of the system will be performed to allow distributed control in different areas of the room. Finally, it will be integrated on a multiobjective optimization problem which will allow controlling users' comfort from thermal, visual and air quality points of view in an efficient way [13]. 


\section{ACKNOWLEDGMENT}

This work has been partially funded by the following project: DPI2014-56364-C2-1-R (financed by the Spanish Ministry of Science and Innovation and EU-ERDF funds). M. Castilla is very grateful to Andalusia Regional Government, Spain, for financing this work through the Program Formación de personal docente e investigador predoctoral en las Universidades Andaluzas, en áreas de conocimiento deficitarias por necesidades docentes (FPDU 2009). This is a programme cofinanced by the European Union through the European Regional Development Fund (ERDF). J.D. Álvarez is a fellow of the Spanish Ramon y Cajal contract program.

\section{REFERENCES}

[1] E. Alcalde, "Potencial de ahorro y eficiencia energética en instalaciones de iluminación (in Spanish)," CIRCE, Tech. Rep., 2012.

[2] S. M. Zanoli and D. Barchiesi, "Thermal and lighting control system with energy saving and users comfort features," in $20^{t h}$ Mediterranean Conference on Control and Automation, MED'12, Barcelona, Spain, 2012.

[3] A. Cziker, M. Chindris, and A. Miron, "Implementation of fuzzy logic in daylighting control," in $11^{\text {th }}$ International Conference on Intelligent Engineering Systems, Budapest, Hungary, 2007.

[4] A. I. Dounis, M. J. Santamouris, and C. C. Lefas, "Building visual comfort control with fuzzy reasoning," Energy Conversion and Management, vol. 34, pp. 17-28, 1993.

[5] A. I. Dounis, P. Tiropanis, A. Argiriou, and A. Diamantis, "Intelligent control system for reconciliation of the energy savings with comfort in buildings using soft computing techniques," Energy and Buildings, vol. 43, pp. 66-74, 2011.

[6] M. Trobec, B. Zupancic, and A. Krainer, "Fuzzy control for the illumination and temperature comfort in a test chamber," Building and Environment, vol. 40, no. 12, pp. 1626-1637, 2005.

[7] L. Ciabattoni, A. Freddi, and G. Ippoliti, "A smart lighting system for industrial and domestic use," in IEEE International Conference on Mechatronics, ICM'13, Vicenza, Italy, 2009.
[8] R. Precup, S. Preitl, M. Radac, E. M. Petriu, C. Dragos, and J. K. Tar, "Experiment-based teaching in advanced control engineering," IEEE Transactions on Education, vol. 54, no. 3, pp. 345-355, 2011.

[9] J. Guasch, R. Forster, F. Ramos, A. Hernández, and N. A. Smith, "Enciclopedia de salud y seguridad en el trabajo: Iluminación (in Spanish)," Organización Internacional del Trabajo. Ministerio de Trabajo y Asuntos Sociales, Tech. Rep., 2001.

[10] CIBSE, Code for Lighting. The Society of Light and Lighting, 2002.

[11] E. 12665, "Light and lighting - Basic terms and criteria for specifying lighting requirements." Brussels: European Commitee for Standardization, 2011.

[12] M. Frontczak and P. Wargocki, "Literature survey on how different factors influence human comfort in indoor environments," Building and Environment, vol. 46, pp. 922-937, 2011.

[13] M. Castilla, J. Álvarez, F. Rodríguez, and M. Berenguel, Comfort Control in Buildings. Springer. Advances Series in Industrial Control, 2014, ISBN: 978-1-4471-6346-6.

[14] E. 12464-1, "Light and lighting - Lighting of work places - Part 1: indoor work places." Brussels: European Commitee for Standardization, 2002.

[15] CIBSE, The SLL Lighting Handbook. The Society of Light and Lighting, 2009.

[16] DIAL GmbH, "http://www.dial.de/DIAL/es/empresa/informacion-deprensa/dialux/dialux-evo-3.html. Last access $27^{\text {th }}$ January, 2015,' 2015.

[17] L. Castillo, "Climate control in inmotic environments (in Spanish)," Master's thesis, University of Almería, 2010.

[18] M. Blanco-Muriel, D. C. Alarcón-Padilla, T. López-Moratalla, and M. Lara-Coira, "Computing the solar vector," Solar Energy, vol. 70, pp. 431-441, 2001.

[19] A. B. Sproul, "Derivation of the solar geometric relationships using vector analysis," Renewable Energy, vol. 32, pp. 1187-1205, 2007.

[20] W. B. Stine and M. Geyer, Power from the sun http://www.powerfromthesun.net/book.html. Last access $07^{\text {th }}$ May, 2015, 2001.

[21] J. Abonyi, Fuzzy Model Identification for Control. Birkhäuser, 2003, ISBN: $0-8176-4238-2$

[22] The Mathworks, Inc., Fuzzy Logic Toolbox. User's Guide, R2014b. 